\title{
KNOPP-TYPE IDENTITIES FOR GENERALIZED MULTIPLE DEDEKIND-TYPE SUMS
}

\author{
Kazuhito KOZUKA
}

(Received 17 October 2013)

\begin{abstract}
In this paper, for any multivariable function with a periodicity and a certain distribution relation, we define a multiple Dedekind-type sum. Then by a combinatorialgeometric method, we study generalizations of Knopp's formula for the classical Dedekind sums. The main theorem contains many of the preceding results concerning generalizations of Knopp's formula.
\end{abstract}

\section{Introduction}

For $h \in \mathbb{Z}$ and $k \in \mathbb{N}$, the classical Dedekind $\operatorname{sum} s(h, k)$ is defined by

$$
s(h, k)=\sum_{\alpha \bmod k}\left(\left(\frac{\alpha}{k}\right)\right)\left(\left(\frac{\alpha h}{k}\right)\right),
$$

where

$$
((x))= \begin{cases}x-[x]-\frac{1}{2} & \text { if } x \notin \mathbb{Z}, \\ 0 & \text { if } x \in \mathbb{Z} .\end{cases}
$$

In this paper, we study generalizations of the following result.

ThEOREM 1.1. For any $N \in \mathbb{N}$, we have

$$
\sum_{\substack{a d=N \\ d>0}} \sum_{b=0}^{d-1} s(a h+k b, d k)=\sigma(N) s(h, k),
$$

where $\sigma(N)=\sum_{\delta \mid N} \delta$ and

$$
\sum_{b=0}^{N-1} s(h+k b, N k)=\sum_{d \mid N} \mu(d) \sigma(N / d) s(d h, k),
$$

where $\mu$ is the Möbius' function. Furthermore (1.2) and (1.3) are equivalent.

The formula (1.2) was obtained by Knopp in [13] by making use of Hecke operators, and the formula (1.3) by Subrahmanyam in [23] by an elementary method. Note that in the special case that $N$ is a prime number, (1.2) was already known to Dedekind [11].

2010 Mathematics Subject Classification: Primary 11F20.

Keywords: Dedekind sums; Knopp's formula.

(C) 2014 Faculty of Mathematics, Kyushu University 
In [19], Parson and Rosen generalized (1.2) to higher-order Dedekind sums defined by substituting Bernoulli functions of higher degree for $((*))$ in $(1.1)$. As for the relation between (1.2) and (1.3), Goldberg showed in [12] that (1.2) is easily deduced from (1.3). In [2], Apostol and Vu generalized the result of Goldberg to Dedekind type sums. In [21], Redmond et al clarified that the generalization of (1.2) to Dedekind type sums and that of (1.3) are equivalent. In [14], [15] and [18], for inhomogeneous Dedekind sums attached to Dirichlet characters, Nagasaka and the author studied generalizations of (1.2) and (1.3) and their equivalence.

In [24], Zheng extended (1.2) to homogeneous Dedekind-type sums and in [4], Beck generalized the result of Zheng to homogeneous multivariable cases.

Recently, by a combinatorial-geometric method, Beck gave new proofs of a well-known reciprocity formula for (1.1) and some of its generalizations $[\mathbf{5}, \mathbf{6}, \mathbf{1 7}]$. The method is based on the work of Carlitz [9] and deeply connected with the theory of lattice points in polytopes [3, 7].

In [16], the author made use of the method and gave a new proof of a generalization of (1.2) obtained in [15].

The purpose of this paper is to extend the method in [16] and generalize Theorem 1.1 to more widely generalized Dedekind-type sums.

Let us give a description of each section.

In Section 2, for a multivariable function having a periodicity and a certain distribution relation attached to Dirichlet characters, we define a multiple Dedekind-type sum and show that the sum contains some of the generalized Dedekind sums defined by means of Bernoulli functions. Then, we state the main theorem of this paper.

As in [16], in order to provide a good overview, we first prove the main theorem for a special simple case in Section 3. A complete proof for the general case is given in Section 4.

\section{Definitions and the main theorem}

Let $R$ be a ring extension of $\mathbb{Q}$ containing all of the roots of unity. Let $m, n \in \mathbb{N}$ and let $\varphi_{1}, \ldots, \varphi_{m+n}$ be Dirichlet characters (not necessarily primitive) defined modulo $N_{\varphi_{1}}, \ldots, N_{\varphi_{m+n}}$, respectively. Consider a map

$$
F: \mathbb{Q}^{m+n} \rightarrow R
$$

satisfying the following conditions (i) and (ii).

(i) For any $\boldsymbol{x} \in \mathbb{Q}^{m+n}$ and $\boldsymbol{l} \in \mathbb{Z}^{m+n}$, we have

$$
F(x+l)=F(x) .
$$

(ii) For each $1 \leq \rho \leq m+n$, there is a constant $v_{\rho} \in \mathbb{Z}$ such that

$$
\begin{aligned}
& \sum_{\lambda \bmod M} F\left(x_{1}, \ldots, x_{\rho-1}, x_{\rho}+\frac{\lambda}{M}, x_{\rho+1}, \ldots, x_{m+n}\right) \\
& \quad=M^{-v_{\rho}} \varphi_{\rho}^{-1}(M) F\left(x_{1}, \ldots, x_{\rho-1}, M x_{\rho}, x_{\rho+1}, \ldots, x_{m+n}\right)
\end{aligned}
$$

holds for any $M \in \mathbb{N}$ with $\operatorname{gcd}\left\{M, N_{\varphi_{\rho}}\right\}=1$. 
By condition (i) we can also regard $F$ as a map from $(\mathbb{Q} / \mathbb{Z})^{m+n}$ to $R$.

Let $H$ be an $m \times n$ matrix with components in $\mathbb{Z}$. In what follows, we consider any $\boldsymbol{x} \in$ $\mathbb{Q}^{m}$ as a row vector, so that $\boldsymbol{x} H$ is defined in $\mathbb{Q}^{n}$ and $F(\boldsymbol{x} \boldsymbol{x} H)$ in $R$. Let $\boldsymbol{k}=\left(k_{1}, \ldots, k_{m}\right) \in$ $\mathbb{N}^{m}$ and let $\mathcal{R}_{m}(\boldsymbol{k}) \subset \mathbb{Z}^{m}$ denote an arbitrarily fixed complete set of representatives of the residue class group $\left(\mathbb{Z} / k_{1} \mathbb{Z}\right) \times \cdots \times\left(\mathbb{Z} / k_{m} \mathbb{Z}\right)$. For $\boldsymbol{x}=\left(x_{1}, \ldots, x_{m}\right) \in \mathbb{Q}^{m}$, we put

$$
\frac{\boldsymbol{x}}{\boldsymbol{k}}=\left(\frac{x_{1}}{k_{1}}, \ldots, \frac{x_{m}}{k_{m}}\right) \text {. }
$$

In what follows we suppose that $\operatorname{gcd}\left\{k_{i}, N_{\varphi_{i}}\right\}=\operatorname{gcd}\left\{k_{i}, N_{\varphi_{m+j}}\right\}=1$ for all $1 \leq i \leq m$ and $1 \leq j \leq n$. We now define a generalized Dedekind-type sum for $F, H$ and $\boldsymbol{k}$ as

$$
\mathcal{S}(F: H, \boldsymbol{k})=\sum_{\boldsymbol{x} \in \mathcal{R}_{m}(\boldsymbol{k})} F\left(\frac{\boldsymbol{x}}{\boldsymbol{k}} \frac{\boldsymbol{x}}{\boldsymbol{k}} H\right) .
$$

In order to show some examples, we recall some definitions and basic properties concerning Bernoulli functions. As usual, we define the $p$ th Bernoulli polynomial $B_{p}(X)$ by

$$
\frac{t e^{t X}}{e^{t}-1}=\sum_{p=0}^{\infty} B_{p}(X) \frac{t^{p}}{p !}
$$

and put $B_{p}=B_{p}(0)$, the $p$ th Bernoulli number. For any $x \in \mathbb{Q}$, we write $x=[x]+\{x\}$ with $[x] \in \mathbb{Z}$ and $0 \leq\{x\}<1$ and define the $p$ th Bernoulli function as $\tilde{B}_{p}(x)=B_{p}(\{x\})$, which is periodic of period one and satisfies a distribution relation such as

$$
\sum_{\lambda \bmod M} \tilde{B}_{p}\left(x+\frac{\lambda}{M}\right)=M^{-p+1} \tilde{B}_{p}(M x)
$$

for any $M \in \mathbb{N}$ and $x \in \mathbb{Q}$ (Raabe's theorem [20]). Note that $((x))=\tilde{B}_{1}(x)$ or zero according as $x \notin \mathbb{Z}$ or $x \in \mathbb{Z}$.

For any primitive Dirichlet character $\chi$, we denote by $f_{\chi}$ the conductor of $\chi$. For any $x \in \mathbb{Q}$ with denominator relatively prime to $f_{\chi}$, we can define the value $\chi(x)$ by multiplicativity. As in [22], we define the twisted $p$ th Bernoulli function $\tilde{B}_{p, \chi}(x)$ by

$$
\sum_{a=0}^{f_{\chi}-1} \frac{\chi(\{x\}+a) t e^{(\{x\}+a) t}}{e^{f_{\chi} t}-1}=\sum_{p=0}^{\infty} \tilde{B}_{p, \chi}(x) \frac{t^{p}}{p !},
$$

or, equivalently,

$$
\tilde{B}_{p, \chi}(x)=f_{\chi}^{p-1} \sum_{a \bmod f_{\chi}} \chi(x+a) \tilde{B}_{p}\left(\frac{x+a}{f_{\chi}}\right)
$$

(cf. p. 301 of [22]). Unless the denominator of $x \in \mathbb{Q}$ is relatively prime to $f_{\chi}$, we put $\tilde{B}_{p, \chi}(x)=0$. Then, it is easily verified that $\tilde{B}_{p, \chi}(x)$ is also periodic of period one and that the distribution relation (2.4) is extended as

$$
\sum_{\lambda \bmod M} \tilde{B}_{p, \chi}\left(x+\frac{\lambda}{M}\right)=M^{-p+1} \chi^{-1}(M) \tilde{B}_{p, \chi}(M x)
$$

for any $M \in \mathbb{N}$ with $\operatorname{gcd}\left\{M, f_{\chi}\right\}=1$ and any $x \in \mathbb{Q}$.

On the basis of the periodicity and the distribution relations as above, let us show some examples of (2.3). 
Example 1. The classical Dedekind sum $s(h, k)$ is the sum (2.3) in the case that $m=n$ $=1, F(x, y)=((x))((y)), H=(h)$, and $\boldsymbol{k}=(k)$.

Example 2. Let $m=n=1, H=(h)$ and $\boldsymbol{k}=(k)$. For $p, q \in \mathbb{Z}_{\geq 0}$ and primitive Dirichlet characters $\chi$ and $\psi$, put $F(x, y)=\tilde{B}_{p, \chi}(x) \tilde{B}_{q, \psi}(y)$. Then

$$
\mathcal{S}(F: H, \boldsymbol{k})=\sum_{\alpha \bmod k} \tilde{B}_{p, \chi}\left(\frac{\alpha}{k}\right) \tilde{B}_{q, \psi}\left(\frac{\alpha h}{k}\right),
$$

which is a higher-order Dedekind sum attached to $\chi$ and $\psi$. In the case of $p=1$ and $\chi=\psi=1$, the sum (2.5) is essentially the same as (1.3) in [1] and the generalization of (1.2) was studied in [19]. The case where $\chi=1$ appeared in [22]. In [14], the author studied a generalizations of Theorem 1.1 for (2.5).

Example 3. Let $m=1, n=2, H=\left(h_{1}, h_{2}\right)$ and $\boldsymbol{k}=(k)$. For $q_{1}, q_{2} \in \mathbb{Z}_{\geq 0}$, put $F\left(x, y_{1}, y_{2}\right)$ $=\tilde{B}_{q_{1}}\left(y_{1}\right) \tilde{B}_{q_{2}}\left(y_{2}\right)$ (which is constant with respect to $x$ ). Then

$$
S(F: H, \boldsymbol{k})=\sum_{\alpha \bmod k} \tilde{B}_{q_{1}}\left(\frac{\alpha h_{1}}{k}\right) \tilde{B}_{q_{2}}\left(\frac{\alpha h_{2}}{k}\right),
$$

which is what is called a homogeneous Dedekind sum. In [24], Zheng studied an extension of (1.2) for (2.6).

Example 4. As a generalization of (2.6), we can consider the case where $m=1$ and in addition to conditions (2.1) and (2.2), $F\left(x_{1}, y_{1}, \ldots, y_{n}\right)$ is constant with respect to $x_{1}$. Put $F_{1}\left(y_{1}, \ldots, y_{n}\right)=F\left(x_{1}, y_{1}, \ldots, y_{n}\right)$ for any $x_{1} \in \mathbb{Q}$. Then for $H=\left(h_{1}, \ldots, h_{n}\right)$ and $\boldsymbol{k}=(k)$, we have

$$
S(F: H, \boldsymbol{k})=\sum_{\alpha \bmod k} F_{1}\left(\frac{\alpha h_{1}}{k}, \ldots, \frac{\alpha h_{n}}{k}\right) .
$$

This includes the sum defined as in Definition 2 of Beck [4].

Example 5. Let

$$
n=1, \quad H=\left(\begin{array}{c}
h_{1} \\
\vdots \\
h_{m}
\end{array}\right), p_{1}, \ldots, p_{m}, q \in \mathbb{Z}_{\geq 0}
$$

and let $\varphi_{1}, \ldots, \varphi_{m}, \psi$ be primitive Dirichlet characters. Put $F\left(x_{1}, \ldots, x_{m}, y\right)=$ $\left(\prod_{i=1}^{m} \tilde{B}_{p_{i}, \varphi_{i}}\left(x_{i}\right)\right) \tilde{B}_{q, \psi}(y)$. Then

$$
S(F: H, \boldsymbol{k})=\sum_{\alpha_{i} \bmod k_{i}(1 \leq i \leq m)}\left(\prod_{i=1}^{m} \tilde{B}_{p_{i}, \varphi_{i}}\left(\frac{\alpha_{i}}{k_{i}}\right)\right) \tilde{B}_{q, \psi}\left(\frac{\alpha_{1} h_{1}}{k_{1}}+\cdots+\frac{\alpha_{m} h_{m}}{k_{m}}\right) .
$$

The special case of this was studied by Carlitz in [8] and [10] with rather modified forms. In [15] and [16], under an additional condition that $k_{1}=\cdots=k_{m}$, the author studied a generalization of Theorem 1.1.

In what follows, we write $\psi_{j}$ and $v_{j}^{\prime}$ for $\varphi_{m+j}$ and $v_{m+j}$, respectively, for $1 \leq j \leq n$. Put

$$
\varphi=\prod_{i=1}^{m} \varphi_{i}, \quad \psi=\prod_{j=1}^{n} \psi_{j}, \quad v=\sum_{i=1}^{m} v_{i} \quad \text { and } \quad v^{\prime}=\sum_{j=1}^{n} v_{j}^{\prime}
$$


For any Dirichlet character $\chi$ and any $l, N \in \mathbb{N}$, we put

$$
\sigma_{l, \chi}(N)=\sum_{\delta \mid N} \chi(\delta) \delta^{l}
$$

For any $d \in \mathbb{N}$, let $\mathcal{I}_{d}$ be a set of $m \times n$ matrices such as

$$
\mathcal{I}_{d}=\left\{B=\left(b_{i j}\right)_{\substack{1 \leq i \leq m \\ 1 \leq j \leq n}} \mid b_{i j} \in \mathbb{Z}, \quad 0 \leq b_{i j} \leq d-1 \text { for all } 1 \leq i \leq m \text { and } 1 \leq j \leq n\right\} .
$$

For $\boldsymbol{k}=\left(k_{1}, \ldots, k_{m}\right)$ and $B \in \mathcal{I}_{d}$, we put

$$
\boldsymbol{k} \circ B=\left(\begin{array}{ccc}
k_{1} & & 0 \\
& \ddots & \\
0 & & k_{m}
\end{array}\right) B,
$$

which is a matrix obtained from $B$ by multiplying the $i$ th row by $k_{i}$ for each $1 \leq i \leq m$. Now we state our main theorem.

Theorem 2.1. Let $N \in \mathbb{N}$ with $\operatorname{gcd}\left\{N, N_{\varphi_{i}}\right\}=\operatorname{gcd}\left\{N, N_{\psi_{j}}\right\}=1$ for all $1 \leq i \leq m$ and $1 \leq j \leq n$. Then we have

$$
\begin{aligned}
& N^{\nu} \varphi(N) \sum_{\substack{a d=N \\
d>0}} \sum_{B \in \mathcal{I}_{d}} d^{\nu^{\prime}-(m-1) n} \psi(d) \mathcal{S}(F: a H+\boldsymbol{k} \circ B, d \boldsymbol{k}) \\
& \quad=\sigma_{\nu+\nu^{\prime}+n, \varphi \psi}(N) \mathcal{S}(F: H, \boldsymbol{k})
\end{aligned}
$$

and

$$
\begin{aligned}
N^{v+v^{\prime}-(m-1) n}(\varphi \psi)(N) \sum_{B \in I_{N}} \mathcal{S}(F: H+\boldsymbol{k} \circ B, N \boldsymbol{k}) \\
=\sum_{d \mid N} \mu(d) d^{v} \varphi(d) \sigma_{v+v^{\prime}+n, \varphi \psi}(N / d) \mathcal{S}(F: d H, \boldsymbol{k}) .
\end{aligned}
$$

Furthermore (2.8) and (2.9) are equivalent.

\section{Proof of Theorem 2.1 in a special case}

In this section, we consider the sum (2.3) in the special case where $m=n=1$ and $\varphi_{1}=\psi_{1}=$ 1. In this case, the conditions (2.1) and (2.2) for the map $F: \mathbb{Q}^{2} \rightarrow R$ in the previous section reduce to the following (3.1) and (3.2), respectively:

$$
\begin{aligned}
F\left(x+l_{1}, y+l_{2}\right) & =F(x, y) \quad \text { for any } x, y \in \mathbb{Q} \text { and } l_{1}, l_{2} \in \mathbb{Z} . \\
\sum_{\lambda \bmod M} F\left(x+\frac{\lambda}{M}, y\right) & =M^{-v} F(M x, y) \text { and } \\
\sum_{\lambda \bmod M} F\left(x, y+\frac{\lambda}{M}\right) & =M^{-v^{\prime}} F(x, M y) \text { for any } x, y \in \mathbb{Q} \text { and } M \in \mathbb{N} .
\end{aligned}
$$

For $h \in \mathbb{Z}$ and $k \in \mathbb{N}$, we put

$$
s(F: h, k)=\sum_{\alpha \bmod k} F\left(\frac{\alpha}{k}, \frac{\alpha h}{k}\right) .
$$


For $l, N \in \mathbb{N}$, we put $\sigma_{l}(N)=\sum_{\delta \mid N} \delta^{l}$. Then the formulas (2.8) and (2.9) in Theorem 2.1 reduce to the following (3.4) and (3.5), respectively:

$$
\begin{gathered}
N^{v} \sum_{\substack{a d=N \\
d>0}} \sum_{b=0}^{d-1} d^{v^{\prime}} s(F: a h+k b, d k)=\sigma_{v+v^{\prime}+1}(N) s(F: h, k), \\
N^{v+v^{\prime}} \sum_{b=0}^{N-1} s(F: h+k b, N k)=\sum_{d \mid N} \mu(d) d^{v} \sigma_{v+v^{\prime}+1}(N / d) s(F: d h, k) .
\end{gathered}
$$

The purpose of this section is to prove (3.4) and (3.5) and their equivalence. In the first place, we prepare some lemmas.

LEMMA 3.1. For any $c \in \mathbb{N}$ we have

$$
s(F: c h, c k)=c^{-v} s(F: h, k) .
$$

Proof. Let $\mathcal{R}(k) \subset \mathbb{Z}$ (respectively $\mathcal{R}(c) \subset \mathbb{Z}$ ) be an arbitrarily fixed complete system of representatives of the residue class group $\mathbb{Z} / k \mathbb{Z}$ (respectively $\mathbb{Z} / c \mathbb{Z}$ ). Then the set $\{\alpha+$ $k l \mid \alpha \in \mathcal{R}(k), l \in \mathcal{R}(c)\}$ is a complete system of representatives of the residue class group $\mathbb{Z} / c k \mathbb{Z}$. It follows from (3.1), (3.2) and (3.3) that

$$
\begin{aligned}
s(F: c h, c k) & =\sum_{\alpha \in \mathcal{R}(k)} \sum_{l \in \mathcal{R}(c)} F\left(\frac{\alpha+k l}{c k}, \frac{(\alpha+k l) c h}{c k}\right) \\
& =\sum_{\alpha \in \mathcal{R}(k)} \sum_{l \in \mathcal{R}(c)} F\left(\frac{\alpha}{c k}+\frac{l}{c}, \frac{\alpha h}{k}\right) \\
& =\sum_{\alpha \in \mathcal{R}(k)} c^{-v} F\left(\frac{\alpha}{k}, \frac{\alpha h}{k}\right)=c^{-v} s(F: h, k) .
\end{aligned}
$$

This completes the proof.

In [16], we introduced an additive subgroup of $\mathbb{Z}^{2}$ such as

$$
\mathcal{A}(a, d: b)=(a,-b) \mathbb{Z}+(0, d) \mathbb{Z}
$$

for $a, d \in \mathbb{N}$ and $b \in \mathbb{Z}$, and as a necessary and sufficient condition for $\left(l_{1}, l_{2}\right) \in \mathbb{Z}^{2}$ to be in $\mathcal{A}(a, d: b)$, we proved the following.

LeMmA 3.2. Let $N=$ ad with $a, d \in \mathbb{N}$ and $b \in \mathbb{Z}$ and let $\left(l_{1}, l_{2}\right) \in \mathbb{Z}^{2}$. Put $d_{1}=$ $\operatorname{gcd}\left\{l_{1}, N\right\}, d_{2}=\operatorname{gcd}\left\{l_{1}, l_{2}, N\right\}, l^{\prime}=l_{1} / d_{1}$ and $N^{\prime}=N / d_{1}$. Then, we have $\left(l_{1}, l_{2}\right) \in$ $\mathcal{A}(a, d: b)$ if and only if the following three conditions hold:

(i) $a \mid d_{1}$;

(ii) $\left(d_{1} / a\right) \mid d_{2}$;

(iii) $l^{\prime} b \equiv-\left(a l_{2} / d_{1}\right)\left(\bmod N^{\prime}\right)$.

As a consequence of Lemma 3.2, we extend Corollary 3.3 of [16] as follows.

LEMMA 3.3. Let $\mathcal{F}$ be a map from $\mathbb{Z}^{2}$ to $R$ and suppose that except for finitely many $\left(l_{1}, l_{2}\right) \in \mathbb{Z}^{2}, \mathcal{F}\left(l_{1}, l_{2}\right)=0$. Then for any $N \in \mathbb{N}$, we have

$$
\sum_{\substack{a d=N \\ d>0}} \sum_{b=0}^{d-1} \sum_{\left(l_{1}, l_{2}\right) \in \mathcal{A}(a, d: b)} \mathcal{F}\left(l_{1}, l_{2}\right)=\sum_{\delta \mid N} \delta \sum_{\left(l_{1}, l_{2}\right) \in(\delta \mathbb{Z})^{2}} \mathcal{F}\left(l_{1}, l_{2}\right)
$$


and

$$
\sum_{b=0}^{N-1} \sum_{\left(l_{1}, l_{2}\right) \in \mathcal{A}(1, N: b)} \mathcal{F}\left(l_{1}, l_{2}\right)=\sum_{d \mid N} \mu(d) \sum_{\delta \mid(N / d)} \delta \sum_{\left(l_{1}, l_{2}\right) \in d \delta \mathbb{Z} \times \delta \mathbb{Z}} \mathcal{F}\left(l_{1}, l_{2}\right) .
$$

Furthermore (3.6) and (3.7) are equivalent.

Proof. We use the same notation as in Lemma 3.2. Note that $\operatorname{gcd}\left\{l^{\prime}, N^{\prime}\right\}=1$. Suppose that $a$ satisfies conditions (i) and (ii) in Lemma 3.2. Then condition (iii) shows that

$$
\sharp\left\{b \in \mathbb{Z} \mid 0 \leq b \leq d-1,\left(l_{1}, l_{2}\right) \in \mathcal{A}(a, d: b)\right\}=\frac{d}{N^{\prime}}=\frac{d d_{1}}{N}=\frac{d_{1}}{a} .
$$

This shows that for any fixed $\left(l_{1}, l_{2}\right) \in \mathbb{Z}^{2}$, the coefficient of $\mathcal{F}\left(l_{1}, l_{2}\right)$ in the left-hand side of (3.6) is $\sum_{a\left|d_{1},\left(d_{1} / a\right)\right| d_{2}} d_{1} / a$. By putting $\delta=d_{1} / a$, this coefficient is equal to $\sum_{\delta \mid d_{2}} \delta$, which is just the coefficient of $\mathcal{F}\left(l_{1}, l_{2}\right)$ in the right-hand side of (3.6). This proves (3.6).

Next, by considering the case of $a=1$ and $d=N$ in Lemma 3.2, a similar argument as in the above proof of (3.6) shows that for any fixed $\left(l_{1}, l_{2}\right) \in \mathbb{Z}^{2}$, the coefficient of $\mathcal{F}\left(l_{1}, l_{2}\right)$ in the left-hand side of (3.7) is $d_{1}$ or zero according to whether $d_{1}=d_{2}$ or $d_{1}>d_{2}$. As for the right-hand side of (3.7), we have $\left(l_{1}, l_{2}\right) \in d \delta \mathbb{Z} \times \delta \mathbb{Z}$ for $d \mid N$ and $\delta \mid(N / d)$ if and only if $d \delta \mid d_{1}$ and $\delta \mid d_{2}$. Hence, the coefficient of $\mathcal{F}\left(l_{1}, l_{2}\right)$ in the right-hand side of (3.7) is $\sum_{\delta \mid d_{2}} \sum_{\delta d \mid d_{1}} \delta \mu(d)=\sum_{\delta \mid d_{2}} \delta \sum_{d \mid\left(d_{1} / \delta\right)} \mu(d)$. Recall that, in general, we have $\sum_{d \mid n} \mu(d)=$ 1 or 0 according to whether $n=1$ or $n>1$. It follows that $\sum_{\delta \mid d_{2}} \delta \sum_{d \mid\left(d_{1} / \delta\right)} \mu(d)=d_{1}$ or 0 according to whether $d_{1}=d_{2}$ or $d_{1}>d_{2}$. Thus, we obtain (3.7).

In order to prove the equivalence between (3.6) and (3.7), we put $\mathcal{F}_{1}\left(l_{1}, l_{2}\right)=\mathcal{F}\left(N l_{1}, l_{2}\right)$ for $\left(l_{1}, l_{2}\right) \in(1 / N) \mathbb{Z} \times \mathbb{Z}$. Note that if $N=a d$ with $a, d \in \mathbb{N}$, the map from $\mathbb{Z}^{2}$ to $\mathbb{Z}^{2}$ defined by mapping $\left(l_{1}, l_{2}\right) \in \mathbb{Z}^{2}$ onto $\left(a l_{1}, l_{2}\right)=\left((N / d) l_{1}, l_{2}\right) \in \mathbb{Z}^{2}$ gives a bijection from $\mathcal{A}(1, d: b)$ to $\mathcal{A}(a, d: b)$. Hence, (3.6) is expressed as

$$
\sum_{d \mid N} \sum_{b=0}^{d-1} \sum_{\left(l_{1}, l_{2}\right) \in \mathcal{A}(1, d: b)} \mathcal{F}_{1}\left(l_{1} / d, l_{2}\right)=\sum_{\delta \mid N} \delta \sum_{\left(l_{1}, l_{2}\right) \in(\delta \mathbb{Z})^{2}} \mathcal{F}_{1}\left(l_{1} / N, l_{2}\right) .
$$

By the Möbius inversion formula, this is equivalent to

$$
\sum_{b=0}^{N-1} \sum_{\left(l_{1}, l_{2}\right) \in \mathcal{A}(1, N: b)} \mathcal{F}_{1}\left(l_{1} / N, l_{2}\right)=\sum_{d \mid N} \mu(d) \sum_{\delta \mid(N / d)} \delta \sum_{\left(l_{1}, l_{2}\right) \in(\delta \mathbb{Z})^{2}} \mathcal{F}_{1}\left(\frac{l_{1}}{N / d}, l_{2}\right)
$$

which is easily transformed into (3.7). This completes the proof.

Now let us proceed to prove (3.4) and (3.5). We consider a half-open parallelogram

$$
\mathcal{P}=\left\{(x, y) \in \mathbb{R}^{2} \mid 0 \leq x<N k, \frac{h x}{k} \leq y<\frac{h x}{k}+N\right\}
$$

and put $D=\mathcal{P} \cap \mathbb{Z}^{2}$, the set of lattice points in $\mathcal{P}$. Let us take

$$
\mathcal{F}\left(l_{1}, l_{2}\right)= \begin{cases}F\left(\frac{l_{1}}{N k}, \frac{1}{N}\left(\frac{l_{1} h}{k}-l_{2}\right)\right) & \text { if }\left(l_{1}, l_{2}\right) \in D, \\ 0 & \text { otherwise }\end{cases}
$$


Let $N=a d$ with $a, d \in \mathbb{N}$. For any $b \in \mathbb{Z}$ with $0 \leq b \leq d-1$ and for any $\left(l_{1}, l_{2}\right) \in$ $\mathcal{A}(a, d: b)$, express

$$
\left(l_{1}, l_{2}\right)=r_{1}(a,-b)+r_{2}(0, d)=\left(a r_{1},-b r_{1}+d r_{2}\right)
$$

with $r_{1}, r_{2} \in \mathbb{Z}$. Then $\left(l_{1}, l_{2}\right) \in D$ holds if and only if $r_{1}$ and $r_{2}$ satisfy

$$
0 \leq a r_{1}<N k \text { and } \frac{a r_{1} h}{k} \leq-b r_{1}+d r_{2}<\frac{a r_{1} h}{k}+N,
$$

namely

$$
0 \leq r_{1}<d k \text { and } 0 \leq r_{2}-\frac{r_{1}}{d k}(a h+k b)<a .
$$

Note that for each $0 \leq r_{1}<d k$, the set of the integers $r_{2}$ satisfying the above condition constitutes a complete system of representatives of the residue class group $\mathbb{Z} / a \mathbb{Z}$. It follows from (3.1) and (3.2) that the left-hand side of (3.6) equals

$$
\begin{aligned}
& \sum_{\substack{a d=N \\
d>0}} \sum_{b=0}^{d-1} \sum_{r_{1}=0}^{d k-1} \sum_{r_{2} \bmod a} F\left(\frac{a r_{1}}{N k}, \frac{1}{N}\left(\frac{a r_{1} h}{k}+b r_{1}-d r_{2}\right)\right) \\
& =\sum_{\substack{a d=N \\
d>0}} \sum_{b=0}^{d-1} \sum_{r_{1}=0}^{d k-1} \sum_{r_{2} \bmod a} F\left(\frac{r_{1}}{d k}, \frac{r_{1}(a h+k b)}{N k}-\frac{r_{2}}{a}\right) \\
& =\sum_{\substack{a d=N \\
d>0}} \sum_{b=0}^{d-1} \sum_{r_{1}=0}^{d k-1} a^{-v^{\prime}} F\left(\frac{r_{1}}{d k}, \frac{r_{1}(a h+k b)}{d k}\right) \\
& =N^{-\nu^{\prime}} \sum_{\substack{a d=N \\
d>0}} d^{\nu^{\prime}} \sum_{b=0}^{d-1} s(F: a h+k b, d k) .
\end{aligned}
$$

On the other hand, the right-hand side of (3.6) equals

$$
\begin{aligned}
& \sum_{\delta \mid N} \delta \sum_{\substack{\left(l_{1}, l_{2}\right) \in \mathbb{Z}^{2} \\
\text { with } \delta\left(l_{1}, l_{2}\right) \in D}} F\left(\frac{\delta l_{1}}{N k}, \frac{1}{N}\left(\frac{\delta l_{1} h}{k}-\delta l_{2}\right)\right) \\
& =\sum_{\delta \mid N} \delta \sum_{l_{1} \bmod N k / \delta} \sum_{l_{2} \bmod N / \delta} F\left(\frac{\delta l_{1}}{N k}, \frac{\delta l_{1} h}{N k}-\frac{\delta l_{2}}{N}\right) \\
& =\sum_{\delta \mid N} \delta \sum_{l_{1} \bmod N k / \delta}(N / \delta)^{-v^{\prime}} F\left(\frac{l_{1}}{N k / \delta}, \frac{l_{1} N h / \delta}{N k / \delta}\right) \\
& =N^{-v^{\prime}} \sum_{\delta \mid N} \delta^{v^{\prime}+1} s(F: N h / \delta, N k / \delta) .
\end{aligned}
$$

By Lemma 3.1, this equals

$$
N^{-v^{\prime}} \sum_{\delta \mid N} \delta^{v^{\prime}+1}(N / \delta)^{-v} s(F: h, k)=N^{-v-v^{\prime}} \sigma_{v+v^{\prime}+1}(N) s(F: h, k) .
$$

Since (3.8) equals (3.9), we obtain (3.4). 
Similarly, the left-hand side of (3.7) equals

$$
\begin{aligned}
& \sum_{b=0}^{N-1} \sum_{\left(l_{1}, l_{2}\right) \in \mathcal{A}(1, N: b) \cap D} F\left(\frac{l_{1}}{N k}, \frac{1}{N}\left(\frac{l_{1} h}{k}-l_{2}\right)\right) \\
& =\sum_{b=0}^{N-1} \sum_{r_{1}=0}^{N k-1} F\left(\frac{r_{1}}{N k}, \frac{1}{N}\left(\frac{r_{1} h}{k}+b r_{1}\right)\right)=\sum_{b=0}^{N-1} \sum_{r_{1}=0}^{N k-1} F\left(\frac{r_{1}}{N k}, \frac{r_{1}}{N k}(h+k b)\right) \\
& =\sum_{b=0}^{N-1} s(F: h+k b, N k) .
\end{aligned}
$$

On the other hand, the right-hand side of (3.7) equals

$$
\begin{aligned}
& \sum_{d \mid N} \mu(d) \sum_{\delta \mid(N / d)} \delta \sum_{\substack{\left(l_{1}, l_{2}\right) \in \mathbb{Z}^{2} \\
\left(\delta d l_{1}, \delta l_{2}\right) \in D}} F\left(\frac{\delta d l_{1}}{N k}, \frac{1}{N}\left(\frac{\delta d l_{1} h}{k}-\delta l_{2}\right)\right) \\
& =\sum_{d \mid N} \mu(d) \sum_{\delta \mid(N / d)} \delta \sum_{l_{1} \bmod N k /(\delta d)} \sum_{l_{2} \bmod N / \delta} F\left(\frac{\delta d l_{1}}{N k}, \frac{\delta d l_{1} h}{N k}-\frac{\delta l_{2}}{N}\right) \\
& =\sum_{d \mid N} \mu(d) \sum_{\delta \mid(N / d)} \delta \sum_{l_{1} \bmod N k /(\delta d)}(N / \delta)^{-v^{\prime}} F\left(\frac{l_{1}}{N k /(\delta d)}, \frac{l_{1} N h / \delta}{N k /(\delta d)}\right) \\
& =N^{-v^{\prime}} \sum_{d \mid N} \mu(d) \sum_{\delta \mid(N / d)} \delta^{v^{\prime}+1} s(F: N h / \delta, N k /(\delta d)) \\
& =N^{-v^{\prime}} \sum_{d \mid N} \mu(d) \sum_{\delta \mid(N / d)} \delta^{v^{\prime}+1}(N / \delta d)^{-v} s(F: d h, k) \text { by Lemma 3.1 } \\
& =N^{-v-v^{\prime}} \sum_{d \mid N} \mu(d) d^{v} \sigma_{v+v^{\prime}+1}(N / d) s(F: d h, k) .
\end{aligned}
$$

Since (3.10) equals (3.11), we obtain (3.5).

Now let us prove the equivalence between (3.4) and (3.5). We first note that (3.4) is equivalent to

$$
\sum_{\substack{a d=N \\ d>0}} \sum_{b=0}^{d-1} d^{v+v^{\prime}} s\left(F: h+d k b, d^{2} k\right)=\sigma_{v+v^{\prime}+1}(N) s(F: h, N k) .
$$

In fact, replacing $k$ by $N k$ in (3.4) and making use of Lemma 3.1, we obtain (3.12) from (3.4). Conversely, (3.4) is deduced from (3.12) by replacing $h$ by $N h$ and making use of Lemma 3.1. Similarly (3.5) is equivalent to

$$
N^{v+v^{\prime}} \sum_{b=0}^{N-1} s\left(F: h+N k b, N^{2} k\right)=\sum_{d \mid N} \mu(d) \sigma_{v+v^{\prime}+1}(N / d) s(F: h, N k / d) .
$$

Then the equivalence between (3.12) and (3.13) is derived from the Möbius inversion formula. This completes the proof.

As special cases, formula (3.4) includes (1.2) of [13], Theorem 3.4 of [19] and Theorem 2 of [2]. Formula (3.5) includes Theorem 3 of [23] and Theorem 1 of [2]. The equivalence between (3.4) and (3.5) includes Theorem 5 of [21]. 


\section{Proof of Theorem 2.1 in the general case}

In this section, we extend the method of the previous section and prove our main theorem completely. We use the same notation as in Section 2.

In addition to $\mathcal{R}_{m}(\boldsymbol{k})$ defined in Section 2 , we denote by $\mathcal{R}_{m}(c)$ for $c \in \mathbb{N}$ an arbitrarily fixed complete set of representatives of the residue class group $(\mathbb{Z} / c \mathbb{Z})^{m}$.

LEMMA 4.1. If $c \in \mathbb{N}$ with $\operatorname{gcd}\left\{c, N_{\varphi_{i}}\right\}=\operatorname{gcd}\left\{c, N_{\psi_{j}}\right\}=1$ for all $1 \leq i \leq m$ and $1 \leq j \leq n$, then we have

$$
\mathcal{S}(F: c H, c \boldsymbol{k})=c^{-v} \varphi^{-1}(c) \mathcal{S}(F: H, \boldsymbol{k}) .
$$

Proof. For $\boldsymbol{k}=\left(k_{1}, \ldots, k_{m}\right), \boldsymbol{l}=\left(l_{1}, \ldots, l_{m}\right) \in \mathbb{Q}^{m}$, we put $\boldsymbol{k} * \boldsymbol{l}=\left(k_{1} l_{1}, \ldots, k_{m} l_{m}\right)$. Then the set $\left\{\boldsymbol{x}+\boldsymbol{k} * \boldsymbol{l} \mid \boldsymbol{x} \in \mathcal{R}_{m}(\boldsymbol{k}), \boldsymbol{l} \in \mathcal{R}_{m}(c)\right\}$ is a complete set of representatives of the residue class group $\left(\mathbb{Z} / c k_{1} \mathbb{Z}\right) \times \cdots \times\left(\mathbb{Z} / c k_{m} \mathbb{Z}\right)$. Hence, by definition $(2.3)$ and by applying (2.1) and (2.2) repeatedly, we have

$$
\begin{aligned}
\mathcal{S}(F: c H, c \boldsymbol{k}) & =\sum_{\boldsymbol{x} \in \mathcal{R}_{m}(\boldsymbol{k})} \sum_{\boldsymbol{l} \in \mathcal{R}_{m}(c)} F\left(\frac{\boldsymbol{x}+\boldsymbol{k} * \boldsymbol{l}}{c \boldsymbol{k}} \frac{\boldsymbol{x}+\boldsymbol{k} * \boldsymbol{l}}{c \boldsymbol{k}} c H\right) \\
& =\sum_{\boldsymbol{x} \in \mathcal{R}_{m}(\boldsymbol{k})} \sum_{\boldsymbol{l} \in \mathcal{R}_{m}(c)} F\left(\frac{\boldsymbol{x}}{c \boldsymbol{k}}+\frac{\boldsymbol{l}}{c} \frac{\boldsymbol{x}}{\boldsymbol{k}} H\right) \\
& =\sum_{\boldsymbol{x} \in \mathcal{R}_{m}(\boldsymbol{k})} c^{-v} \varphi^{-1}(c) F\left(\frac{\boldsymbol{x}}{\boldsymbol{k}} \frac{\boldsymbol{x}}{\boldsymbol{k}} H\right)=c^{-v} \varphi^{-1}(c) \mathcal{S}(F: H, \boldsymbol{k}) .
\end{aligned}
$$

This completes the proof.

Let $a, d \in \mathbb{N}$ and let $B$ be an $m \times n$ matrix with components in $\mathbb{Z}$. Note that the additive group $\mathcal{A}(a, d: b)$ in the previous section is expressed as

$$
\mathcal{A}(a, d: b)=\left\{\left(r_{1} r_{2}\right)\left(\begin{array}{cc}
a & -b \\
0 & d
\end{array}\right) \mid r_{1}, r_{2} \in \mathbb{Z}\right\} .
$$

Generalizing this, we define an additive subgroup of $\mathbb{Z}^{m+n}$ as

$$
\mathcal{A}(a, d: B)=\left\{\left(\begin{array}{ll}
\boldsymbol{r}_{1} & \boldsymbol{r}_{2}
\end{array}\right)\left(\begin{array}{c|c}
a E_{m} & -B \\
\hline O & d E_{n}
\end{array}\right) \mid \boldsymbol{r}_{1} \in \mathbb{Z}^{m}, \quad \boldsymbol{r}_{2} \in \mathbb{Z}^{n}\right\},
$$

where $E_{m}$ and $E_{n}$ are unit matrices of degrees $m$ and $n$, respectively. Note that in the case of $n=1$, this group coincides with $A(a, d: B)$ in Section 4 of [16]. The next lemma is a generalization of Lemma 3.2 and also of Lemma 4.2 of [16].

LEMmA 4.2. Let $N=$ ad with $a, d \in \mathbb{N}$ and $B$ an $m \times n$ matrix with components in $\mathbb{Z}$. Let $\left(\boldsymbol{l}_{1} \boldsymbol{l}_{2}\right) \in \mathbb{Z}^{m+n}$ with $\boldsymbol{l}_{1}=\left(l_{11}, \ldots, l_{1 m}\right)$ and $\boldsymbol{l}_{2}=\left(l_{21}, \ldots, l_{2 n}\right)$. Put $d_{1}=$ $\operatorname{gcd}\left\{l_{11}, \ldots, l_{1 m}, N\right\}, d_{2}=\operatorname{gcd}\left\{d_{1}, l_{21}, \ldots, l_{2 n}, N\right\}, l_{1}^{\prime}=l_{1} / d_{1}$ and $N^{\prime}=N / d_{1}$. Then, we have $\left(\begin{array}{ll}\boldsymbol{l}_{1} & \boldsymbol{l}_{2}\end{array}\right) \in \mathcal{A}(a, d: B)$ if and only if the following three conditions hold:

(i) $a \mid d_{1}$;

(ii) $\left(d_{1} / a\right) \mid d_{2}$;

(iii) $\boldsymbol{l}_{1}^{\prime} B \equiv-\left(a \boldsymbol{l}_{2} / d_{1}\right)\left(\bmod N^{\prime} \mathbb{Z}^{n}\right)$. 
Proof. Let $\left(\boldsymbol{l}_{1} \boldsymbol{l}_{2}\right) \in \mathcal{A}(a, d: B)$ and express

$$
\left(\begin{array}{ll}
\boldsymbol{l}_{1} & \boldsymbol{l}_{2}
\end{array}\right)=\left(\begin{array}{ll}
\boldsymbol{r}_{1} & \boldsymbol{r}_{2}
\end{array}\right)\left(\begin{array}{c|c}
a E_{m} & -B \\
\hline O & d E_{n}
\end{array}\right)
$$

with $\boldsymbol{r}_{1} \in \mathbb{Z}^{m}$ and $\boldsymbol{r}_{2} \in \mathbb{Z}^{n}$. Then we have $\boldsymbol{l}_{1}=a \boldsymbol{r}_{1}$, which shows the condition (i). Further, we also have $\boldsymbol{l}_{2}=-\boldsymbol{r}_{1} B+d \boldsymbol{r}_{2}$. Multiplying $a / d_{1}$ on both sides, we obtain

$$
\frac{1}{d_{1} / a} \boldsymbol{l}_{2}=-\frac{a}{d_{1}} \boldsymbol{r}_{1} B+\frac{N}{d_{1}} \boldsymbol{r}_{2}=-\boldsymbol{l}_{1}^{\prime} B+N^{\prime} \boldsymbol{r}_{2} \in \mathbb{Z}^{n},
$$

which shows conditions (ii) and (iii). Conversely under conditions (i), (ii) and (iii), we can easily deduce (4.1) with $\boldsymbol{r}_{1} \in \mathbb{Z}^{m}$ and $\boldsymbol{r}_{2} \in \mathbb{Z}^{n}$. This completes the proof.

LEMMA 4.3. Let $\mathcal{F}$ be a map from $\mathbb{Z}^{m+n}$ to $R$ and suppose that except for finitely many $\left(\begin{array}{ll}l_{1} & l_{2}\end{array}\right) \in \mathbb{Z}^{m+n}, \quad \mathcal{F}\left(l_{1} l_{2}\right)=0$. Then for any $N \in \mathbb{N}$, we have

$$
\begin{gathered}
\sum_{\substack{a d=N \\
d>0}} \sum_{B \in \mathcal{I}_{d}} \sum_{\left(\boldsymbol{l}_{1}\right.} \sum_{\left.\boldsymbol{l}_{2}\right) \in \mathcal{A}(a, d: B)} a^{(m-1) n} \mathcal{F}\left(\boldsymbol{l}_{1} \boldsymbol{l}_{2}\right) \\
\left.=N^{(m-1) n} \sum_{\delta \mid N} \delta^{n} \sum_{\left(\boldsymbol{l}_{1}\right.} \sum_{2}\right) \in(\delta \mathbb{Z})^{m+n} \\
\mathcal{F}\left(\boldsymbol{l}_{1} \boldsymbol{l}_{2}\right),
\end{gathered}
$$

and

$$
\begin{aligned}
& \sum_{B \in \mathcal{I}_{N}} \sum_{\left(\boldsymbol{l}_{1}\right.} \sum_{\left.\boldsymbol{l}_{2}\right) \in \mathcal{A}(1, N: B)} \mathcal{F}\left(\boldsymbol{l}_{1} \boldsymbol{l}_{2}\right) \\
& =N^{(m-1) n} \sum_{d \mid N} \mu(d) \sum_{\delta \mid(N / d)} \delta^{n} \sum_{\left(\boldsymbol{l}_{1}\right.} \sum_{\left.\boldsymbol{l}_{2}\right) \in d \delta \mathbb{Z}^{m} \times \delta \mathbb{Z}^{n}} \mathcal{F}\left(\boldsymbol{l}_{1} \boldsymbol{l}_{2}\right) .
\end{aligned}
$$

Furthermore (4.3) and (4.4) are equivalent.

Proof. Let $\boldsymbol{l}_{1} \in \mathbb{Z}^{m}$ and $\boldsymbol{l}_{2} \in \mathbb{Z}^{n}$ and we use the same notation as in Lemma 4.2. Put $\boldsymbol{l}_{1}^{\prime}=$ $\left(l_{11}^{\prime}, \ldots, l_{1 m}^{\prime}\right)$. Let $N=a d$ with $a, d \in \mathbb{N}$ and suppose that $a$ satisfies conditions (i) and (ii). Let us consider the number of $B \in \mathcal{I}_{d}$ satisfying condition (iii). Let $B=\left(b_{i j}\right)_{\substack{1 \leq i \leq m \\ 1 \leq j \leq n}}$ with $0 \leq b_{i j} \leq d-1$. Then condition (iii) means that for each $1 \leq j \leq n$, the transposition of $j$ th column of $B$, namely $\left(b_{1 j}, \ldots, b_{m j}\right)$, is one of the solutions $\left(x_{1}, \ldots, x_{m}\right)$ of the congruence

$$
l_{11}^{\prime} x_{1}+\cdots+l_{1 m}^{\prime} x_{m} \equiv-\frac{a l_{2 j}}{d_{1}} \quad\left(\bmod N^{\prime}\right)
$$

Note that the map from $\mathbb{Z}^{m}$ to $\mathbb{Z}$ defined by mapping $\left(x_{1}, \ldots, x_{m}\right)$ onto $l_{11}^{\prime} x_{1}+$ $\cdots+l_{1 m}^{\prime} x_{m}$ induces a map from $\left(\mathbb{Z} / N^{\prime} \mathbb{Z}\right)^{m}$ to $\mathbb{Z} / N^{\prime} \mathbb{Z}$, which is surjective because $\operatorname{gcd}\left\{l_{11}^{\prime}, \ldots, l_{1 m}^{\prime}, N^{\prime}\right\}=1$. Hence, the number of $m$-tuples $\left(x_{1}, \ldots, x_{m}\right) \bmod N^{\prime} \in$ $\left(\mathbb{Z} / N^{\prime} \mathbb{Z}\right)^{m}$ satisfying (4.4) is $\sharp\left(\mathbb{Z} / N^{\prime} \mathbb{Z}\right)^{m} / \sharp\left(\mathbb{Z} / N^{\prime} \mathbb{Z}\right)=N^{\prime m-1}$. Note that $d / N^{\prime}=d d_{1} / N=$ $d_{1} / a \in \mathbb{N}$ and it follows that for each $1 \leq j \leq n$, the number of $m$-tuples $\left(b_{1 j}, \ldots, b_{m j}\right) \in$ $\mathbb{Z}^{m}$ which satisfies $0 \leq b_{i j} \leq d-1$ and congruence (4.4) by taking $\left(x_{1}, \ldots, x_{m}\right)=$ $\left(b_{1 j}, \ldots, b_{m j}\right)$ is

$$
N^{\prime m-1}\left(\frac{d}{N^{\prime}}\right)^{m}=\frac{d^{m}}{N^{\prime}}=\frac{d^{m-1} d_{1}}{a} .
$$


Hence, the number of $B \in \mathcal{I}_{d}$ satisfying condition (iii) is $\left(d^{m-1} d_{1} / a\right)^{n}$. This shows that for any fixed $\left(\begin{array}{ll}\boldsymbol{l}_{1} & \boldsymbol{l}_{2}\end{array}\right) \in \mathbb{Z}^{m+n}$, the coefficient of $\mathcal{F}\left(\boldsymbol{l}_{1} \boldsymbol{l}_{2}\right)$ in the left-hand side of (4.2) is

$$
\sum_{\substack{a\left|d_{1} \\\left(d_{1} / a\right)\right| d_{2}}} a^{(m-1) n}\left(\frac{d^{m-1} d_{1}}{a}\right)^{n}=N^{(m-1) n} \sum_{\substack{a\left|d_{1} \\\left(d_{1} / a\right)\right| d_{2}}}\left(\frac{d_{1}}{a}\right)^{n} .
$$

By putting $\delta=d_{1} / a$, this coefficient is equal to $N^{(m-1) n} \sum_{\delta \mid d_{2}} \delta^{n}$, which is just the coefficient of $\mathcal{F}\left(\boldsymbol{l}_{1} \boldsymbol{l}_{2}\right)$ in the right-hand side of (4.2). This proves (4.2).

Next, by considering the case of $a=1$ and $d=N$ in Lemma 4.2, similar argument as in the above proof of (4.2) shows that for any fixed $\left(\begin{array}{ll}\boldsymbol{l}_{1} & \boldsymbol{l}_{2}\end{array}\right) \in \mathbb{Z}^{m+n}$, the coefficient of $\mathcal{F}\left(\boldsymbol{l}_{1} \boldsymbol{l}_{2}\right)$ in the left-hand side of (4.3) is $N^{(m-1) n} d_{1}^{n}$ or zero according as $d_{1}=d_{2}$ or $d_{1}>d_{2}$. In the similar way as in the proof of (3.7) of Lemma 3.3, we can also deduce the coefficient in the right-hand side of (4.3) and verify the formula (4.3).

In order to prove the equivalence between (4.2) and (4.3), we put

$$
\mathcal{F}_{1}\left(\boldsymbol{l}_{1} \boldsymbol{l}_{2}\right)=\mathcal{F}\left(\begin{array}{ll}
N \boldsymbol{l}_{1} & \boldsymbol{l}_{2}
\end{array}\right) \quad \text { for }\left(\boldsymbol{l}_{1} \boldsymbol{l}_{2}\right) \in\left(\frac{1}{N} \mathbb{Z}\right)^{m} \times \mathbb{Z}^{n} .
$$

Note that if $N=a d$ with $a, d \in \mathbb{N}$, the map from $\mathbb{Z}^{m+n}$ to $\mathbb{Z}^{m+n}$ defined by mapping $\left(\begin{array}{ll}\boldsymbol{l}_{1} & \boldsymbol{l}_{2}\end{array}\right) \in \mathbb{Z}^{m+n}$ onto $\left(\begin{array}{ll}a \boldsymbol{l}_{1} & \boldsymbol{l}_{2}\end{array}\right)=\left((N / d) \boldsymbol{l}_{1} \boldsymbol{l}_{2}\right)$ gives a bijection from $\mathcal{A}(1, d: B)$ to $\mathcal{A}(a, d: B)$. Hence, (4.2) is expressed as

$$
\begin{gathered}
\sum_{d \mid N} \sum_{B \in \mathcal{I}_{d}} \sum_{\left(\boldsymbol{l}_{1}\right.} \sum_{\left.\boldsymbol{l}_{2}\right) \in \mathcal{A}(1, d: B)}\left(\frac{N}{d}\right)^{(m-1) n} \mathcal{F}_{1}\left(\frac{\boldsymbol{l}_{1}}{d} \boldsymbol{l}_{2}\right) \\
=N^{(m-1) n} \sum_{\delta \mid N} \delta^{n} \sum_{\left(\boldsymbol{l}_{1}\right.} \sum_{\left.\boldsymbol{l}_{2}\right) \in(\delta \mathbb{Z})^{m+n}} \mathcal{F}_{1}\left(\frac{\boldsymbol{l}_{1}}{N} \boldsymbol{l}_{2}\right),
\end{gathered}
$$

namely

$$
\sum_{d \mid N} \sum_{B \in \mathcal{I}_{d}} \sum_{\left(\boldsymbol{l}_{1}\right.} \frac{1}{\left.\boldsymbol{l}_{2}\right) \in \mathcal{A}(1, d: B)} \bar{d}^{(m-1) n} \mathcal{F}_{1}\left(\frac{\boldsymbol{l}_{1}}{d} \boldsymbol{l}_{2}\right)=\sum_{\delta \mid N} \delta^{n} \sum_{\left(\boldsymbol{l}_{1}\right.} \sum_{\left.\boldsymbol{l}_{2}\right) \in(\delta \mathbb{Z})^{m+n}} \mathcal{F}_{1}\left(\frac{\boldsymbol{l}_{1}}{N} \boldsymbol{l}_{2}\right) .
$$

By the Möbius inversion formula, this is equivalent to

$$
\begin{aligned}
& \sum_{B \in \mathcal{I}_{N}} \sum_{\left(\boldsymbol{l}_{1}\right.} \frac{1}{\left.\boldsymbol{l}_{2}\right) \in \mathcal{A}(1, N: B)} \frac{1}{N^{(m-1) n}} \mathcal{F}_{1}\left(\frac{\boldsymbol{l}_{1}}{N} \boldsymbol{l}_{2}\right) \\
& \left.=\sum_{d \mid N} \mu(d) \sum_{\delta \mid(N / d)} \delta^{n} \sum_{\left(\boldsymbol{l}_{1}\right.} \boldsymbol{l}_{2}\right) \in(\delta \mathbb{Z})^{m+n} \\
& \mathcal{F}_{1}\left(\frac{d \boldsymbol{l}_{1}}{N} \boldsymbol{l}_{2}\right),
\end{aligned}
$$

which is easily transformed into (4.3). This completes the proof.

Proof of Theorem 2.1. For $l \in \mathbb{N}$ and $c=\left(c_{1}, \ldots, c_{l}\right) \in \mathbb{N}^{l}$, we put

$$
\mathcal{J}_{l}(\boldsymbol{c})=\left\{\left(x_{1}, \ldots, x_{l}\right) \in \mathbb{R}^{l} \mid 0 \leq x_{i}<c_{i} \text { for each } 1 \leq i \leq l\right\} .
$$

If $\boldsymbol{c}=(c, \ldots, c) \in \mathbb{N}^{l}$, we put $\mathcal{J}_{l}(c)=\mathcal{J}_{l}(\boldsymbol{c})$.

We put

$$
D=\left\{\left(\boldsymbol{l}_{1} \boldsymbol{l}_{2}\right) \in \mathbb{Z}^{m+n} \mid \boldsymbol{l}_{1} \in \mathcal{J}_{m}(N \boldsymbol{k}), \boldsymbol{l}_{2}-\frac{\boldsymbol{l}_{1}}{\boldsymbol{k}} H \in \mathcal{J}_{n}(N)\right\} .
$$


and take

$$
\mathcal{F}\left(\begin{array}{ll}
\boldsymbol{l}_{1} & \boldsymbol{l}_{2}
\end{array}\right)= \begin{cases}F\left(\frac{\boldsymbol{l}_{1}}{N \boldsymbol{k}} \frac{1}{N}\left(\frac{\boldsymbol{l}_{1}}{\boldsymbol{k}} H-\boldsymbol{l}_{2}\right)\right) & \text { if }\left(\boldsymbol{l}_{1} \boldsymbol{l}_{2}\right) \in D, \\
0 & \text { otherwise }\end{cases}
$$

For $N=a d$ with $a, d \in \mathbb{N}$ and $B \in \mathcal{I}_{d}$, let $\left(\boldsymbol{l}_{1} \boldsymbol{l}_{2}\right) \in \mathcal{A}(a, d: B)$ and express

$$
\left(\begin{array}{ll}
\boldsymbol{l}_{1} & \boldsymbol{l}_{2}
\end{array}\right)=\left(\begin{array}{ll}
\boldsymbol{r}_{1} & \boldsymbol{r}_{2}
\end{array}\right)\left(\begin{array}{c|c}
a E_{m} & -B \\
\hline O & d E_{n}
\end{array}\right)=\left(\begin{array}{l}
a \boldsymbol{r}_{1}-\boldsymbol{r}_{1} B+d \boldsymbol{r}_{2}
\end{array}\right)
$$

with $\boldsymbol{r}_{1} \in \mathbb{Z}^{m}$ and $\boldsymbol{r}_{2} \in \mathbb{Z}^{n}$. Then $\left(\boldsymbol{l}_{1} \boldsymbol{l}_{2}\right) \in D$ holds if and only if we have

$$
a \boldsymbol{r}_{1} \in \mathcal{J}_{m}(N \boldsymbol{k}) \quad \text { and } \quad\left(-\boldsymbol{r}_{1} B+d \boldsymbol{r}_{2}\right)-\frac{a \boldsymbol{r}_{1}}{\boldsymbol{k}} H \in \mathcal{J}_{n}(N),
$$

which are equivalent to

$$
\boldsymbol{r}_{1} \in \mathcal{J}_{m}(d \boldsymbol{k}) \quad \text { and } \quad \boldsymbol{r}_{2}-\frac{\boldsymbol{r}_{1}}{d \boldsymbol{k}}(a H+\boldsymbol{k} \circ B) \in \mathcal{J}_{n}(a),
$$

respectively. Note that for each $\boldsymbol{r}_{1} \in \mathcal{J}_{m}(d \boldsymbol{k})$, the set of $\boldsymbol{r}_{2} \in \mathbb{Z}^{n}$ satisfying the above condition constitutes a complete system of representatives of the residue class group $(\mathbb{Z} / a \mathbb{Z})^{n}$. Applying (2.1) and (2.2) repeatedly, and recalling the notation (2.7), we see that the left-hand side of (4.2) equals

$$
\begin{aligned}
& \sum_{\substack{a d=N \\
d>0}} \sum_{B \in \mathcal{I}_{d}} \sum_{\boldsymbol{r}_{1} \in \mathcal{J}_{m}(d \boldsymbol{k}) \cap \mathbb{Z}^{m}} \sum_{\boldsymbol{r}_{2} \in(\mathbb{Z} / a \mathbb{Z})^{n}} a^{(m-1) n} F\left(\frac{a \boldsymbol{r}_{1}}{N \boldsymbol{k}} \frac{\boldsymbol{r}_{1}}{N \boldsymbol{k}}(a H+\boldsymbol{k} \circ B)-\frac{\boldsymbol{r}_{2}}{a}\right) \\
& =\sum_{\substack{a d=N \\
d>0}} \sum_{B \in \mathcal{I}_{d}} \sum_{\boldsymbol{r}_{1} \in \mathcal{J}_{m}(d \boldsymbol{k}) \cap \mathbb{Z}^{m}} a^{(m-1) n} a^{-v^{\prime}} \psi^{-1}(a) F\left(\frac{\boldsymbol{r}_{1}}{d \boldsymbol{k}} \frac{\boldsymbol{r}_{1}}{d \boldsymbol{k}}(a H+\boldsymbol{k} \circ B)\right) \\
& =N^{(m-1) n-v^{\prime}} \psi^{-1}(N) \sum_{\substack{a d=N \\
d>0}} \sum_{B \in \mathcal{I}_{d}} d^{\nu^{\prime}-(m-1) n} \psi(d) \mathcal{S}(F: a H+\boldsymbol{k} \circ B, d \boldsymbol{k}) .
\end{aligned}
$$

On the other hand, the right-hand side of (4.2) equals

$$
\begin{aligned}
N^{(m-1) n} \sum_{\delta \mid N} \delta^{n} \sum_{\substack{\left(\boldsymbol{l}_{1} \boldsymbol{l}_{2}\right) \in \mathbb{Z}^{m} \times \mathbb{Z}^{n} \\
\text { with } \delta\left(\boldsymbol{l}_{1}\right.}} F\left(\frac{\left.\delta \boldsymbol{l}_{1}\right) \in D}{N \boldsymbol{k}} \frac{\delta \boldsymbol{l}_{1}}{N \boldsymbol{k}} H-\frac{\delta \boldsymbol{l}_{2}}{N}\right) \\
=N^{(m-1) n} \sum_{\delta \mid N} \delta^{n} \sum_{\boldsymbol{l}_{1} \in \mathcal{R}_{m}(N \boldsymbol{k} / \delta)} \sum_{\boldsymbol{l}_{2} \in \mathcal{R}_{n}(N / \delta)} F\left(\frac{\delta \boldsymbol{l}_{1}}{N \boldsymbol{k}} \frac{\delta \boldsymbol{l}_{1}}{N \boldsymbol{k}} H-\frac{\delta \boldsymbol{l}_{2}}{N}\right) \\
=N^{(m-1) n} \sum_{\delta \mid N} \delta^{n} \sum_{\boldsymbol{l}_{1} \in \mathcal{R}_{m}(N \boldsymbol{k} / \delta)}(N / \delta)^{-v^{\prime}} \psi^{-1}(N / \delta) F\left(\frac{\boldsymbol{l}_{1}}{(N / \delta) \boldsymbol{k}} \frac{\boldsymbol{l}_{1}}{(N / \delta) \boldsymbol{k}}(N / \delta) H\right) \\
=N^{(m-1) n-v^{\prime}} \psi^{-1}(N) \sum_{\delta \mid N} \delta^{n+v^{\prime}} \psi(\delta) \mathcal{S}(F:(N / \delta) H,(N / \delta) \boldsymbol{k}) \\
=N^{(m-1) n-v^{\prime}} \psi^{-1}(N) \sum_{\delta \mid N} \delta^{n+v^{\prime}} \psi(\delta)(N / \delta)^{-v} \varphi^{-1}(N / \delta) \mathcal{S}(F: H, \boldsymbol{k}) \quad \text { by Lemma 4.1 }
\end{aligned}
$$


Since (4.5) equals (4.6), we obtain (2.8).

Similarly, the left-hand side of (4.3) equals

$$
\begin{aligned}
& \sum_{B \in \mathcal{I}_{N}} \sum_{\boldsymbol{r}_{1} \in \mathcal{J}_{m}(N \boldsymbol{k}) \cap \mathbb{Z}^{m}} F\left(\frac{\boldsymbol{r}_{1}}{N \boldsymbol{k}} \frac{\boldsymbol{r}_{1}}{N \boldsymbol{k}}(H+\boldsymbol{k} \circ B)\right) \\
& =\sum_{B \in \mathcal{I}_{N}} \mathcal{S}(F: H+\boldsymbol{k} \circ B, N \boldsymbol{k}) .
\end{aligned}
$$

The right-hand side of (4.3) equals

$$
\begin{aligned}
& N^{(m-1) n} \sum_{d \mid N} \mu(d) \sum_{\delta \mid(N / d)} \delta^{n} \sum_{\left(\boldsymbol{l}_{1}\right.} \sum_{\left.\boldsymbol{l}_{2}\right) \in \mathbb{Z}^{m+n}} \mathcal{F}\left(\delta d \boldsymbol{l}_{1} \delta \boldsymbol{l}_{2}\right)
\end{aligned}
$$

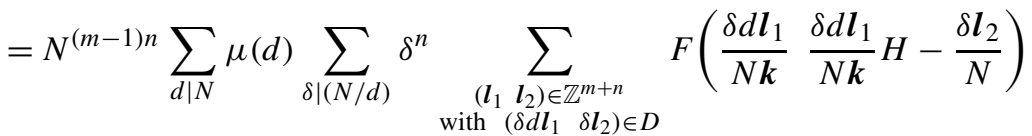

$$
\begin{aligned}
& =N^{(m-1) n} \sum_{d \mid N} \mu(d) \sum_{\delta \mid(N / d)} \delta^{n} \sum_{\boldsymbol{l}_{1} \in \mathcal{J}_{m}(N \boldsymbol{k} /(\delta d)) \cap \mathbb{Z}^{m}} \sum_{\boldsymbol{l}_{2} \in \mathcal{R}_{n}(N / \delta)} F\left(\frac{\delta d \boldsymbol{l}_{1}}{N \boldsymbol{k}} \frac{\delta d \boldsymbol{l}_{1}}{N \boldsymbol{k}} H-\frac{\delta \boldsymbol{l}_{2}}{N}\right) \\
& =N^{(m-1) n} \sum_{d \mid N} \mu(d) \sum_{\delta \mid(N / d)} \delta^{n}(N / \delta)^{-v^{\prime}} \psi^{-1}(N / \delta) \\
& \times \sum_{\boldsymbol{l}_{1} \in \mathcal{R}_{m}(N \boldsymbol{k} /(d \delta))} F\left(\frac{\boldsymbol{l}_{1}}{(N /(\delta d)) \boldsymbol{k}} \frac{\boldsymbol{l}_{1}}{(N /(\delta d)) \boldsymbol{k}}(N / \delta) H\right) \\
& =N^{(m-1) n-v^{\prime}} \psi^{-1}(N) \sum_{d \mid N} \mu(d) \sum_{\delta \mid(N / d)} \delta^{\nu^{\prime}+n} \psi(\delta) \mathcal{S}(F:(N / \delta) H,(N /(\delta d)) \boldsymbol{k}) .
\end{aligned}
$$

By Lemma 4.1, this also equals

$$
N^{(m-1) n-v-v^{\prime}}(\varphi \psi)^{-1}(N) \sum_{d \mid N} \mu(d) d^{\nu} \varphi(d) \sigma_{\nu+\nu^{\prime}+n, \varphi \psi}(N / d) \mathcal{S}(F: d H, \boldsymbol{k})
$$

and thereby we obtain (2.9).

Finally let us prove the equivalence between (2.8) and (2.9). As in the proof of the equivalence between (3.4) and (3.5) in Section 3, we can replace (2.8) (respectively (2.9)) by the following equivalent formula (4.7) (respectively (4.8)) derived from Lemma 4.1:

$$
\begin{aligned}
& \sum_{\substack{a d=N \\
d>0}} \sum_{B \in \mathcal{I}_{d}} d^{v+v^{\prime}-(m-1) n}(\varphi \psi)(d) \mathcal{S}\left(F: H+d \boldsymbol{k} \circ B, d^{2} \boldsymbol{k}\right) \\
& \quad=\sigma_{v+v^{\prime}+n, \varphi \psi}(N) \mathcal{S}(F: H, N \boldsymbol{k}) . \\
& N^{v+v^{\prime}-(m-1) n}(\varphi \psi)(N) \sum_{B \in I_{N}} \mathcal{S}\left(F: H+N \boldsymbol{k} \circ B, N^{2} \boldsymbol{k}\right) \\
& =\sum_{d \mid N} \mu(d) \sigma_{v+v^{\prime}+n, \varphi \psi}(N / d) \mathcal{S}(F: H, N \boldsymbol{k} / d) .
\end{aligned}
$$

Then (4.7) and (4.8) are obviously equivalent by the Möbius inversion formula. This completes the proof. 
As special cases, our main theorem includes Theorems 1, 2 and 3 of [24], Theorem 11 of [4], Theorem 2.3 of [14] and Theorem 4.1 of [15].

\title{
REFERENCES
}

[1] T. M. Apostol. Generalized Dedekind sums and transformation formulae of certain Lambert series. Duke Math. J. 17 (1950), 147-157.

[2] T. M. Apostol and T. H. Vu. Identities for sums of Dedekind type. J. Number Theory 14 (1982), 391-396.

[3] A. Barvinok and J. E. Pommersheim. An algorithmic theory of lattice points in polyhedra. New perspectives in algebraic combinatorics (Berkeley, CA, 1996-97), 91-147.

[4] M. Beck. Dedekind cotangent sums. Acta Arith. 109 (2003), 109-130.

[5] M. Beck. Geometric proofs of polynomial reciprocity laws of Carlitz, Berndt and Dieter. Diophantine analysis and related field 2006, Sem. Math. Sci. Keio Univ. 35 (2006), 11-18.

[6] M. Beck, C. Haase and A. R. Matthews. Dedekind-Carlitz polynomials as lattice-point enumerators in rational polyhedra. Math. Ann. 341 (2008), 945-961.

[7] M. Beck and S. Robins. Computing the Continuous Discretely: Integerpoint Enumeration in Polyhedra (Undergraduate Texts in Mathematics). Springer, New York, 2007.

[8] L. Carlitz. A note on generalized Dedekind sums. Duke. Math. J. 21 (1954), 399-403.

[9] L. Carlitz. Some polynomials associated with Dedekind sums. Acta Math. Soc. Hungury 26 (1975), 311319.

[10] L. Carlitz. Many-term relations for multiple Dedekind sums. Indian J. Math. 20 (1978), 77-89.

[11] R. Dedekind. Erläuterungen zu zwei Fragmenten von Riemann. Gesammelte mathematische Werke, Bd. I. S, Vieweg, Braunschweig, 1930, pp. 159-173.

[12] L. A. Goldberg. An elementary proof of the Petersson-Knopp theorem on Dedekind sums. J. Number Theory 12 (1980), 541-542.

[13] M. I. Knopp. Hecke operators and an identity for the Dedekind sums. J. Number Theory 12 (1980), 2-9.

[14] K. Kozuka. Dedekind type sums attached to Dirichlet characters. Kyushu J. Math. 58 (2004), 1-24.

[15] K. Kozuka. Some identities for multiple Dedekind sums attached to Dirichlet characters. Acta Arith. 146 (2011), 103-114.

[16] K. Kozuka. Combinatorial-geometric viewpoint of Knopp's formula for Dedekind sums. Funct. Approx. 46 (2012), 79-89.

[17] A. R. Matthews. A Geometric Approach to Carlitz-Dedekind sums. San Francisco State University, San Francisco, CA, 2007.

[18] C. Nagasaka. Dedekind type sums and Hecke operators. Acta Arith. 44 (1984), 207-214.

[19] L. A. Parson and K. H. Rosen. Hecke operators and Lambert series. Math. Scand. 49 (1981), 5-14.

[20] J. L. Raabe. Zurückführung einiger Summen und bestimmten Integrale auf die Jacob-Bernoullische Funktion. J. Reine Angew. Math. 42 (1851), 348-367.

[21] D. Redmond, R. S. Rao and R. Sivaramakrishanan. On Dedekind sums and analogs. Tsukuba J. Math. 12 (1988), 371-389.

[22] C. Snyder. $p$-adic interpolation of Dedekind sums. Bull. Aust. Math. Soc. 38 (1988), 293-301.

[23] P. Subrahmanyam. On sums involving the integer part of $x$. Math. Student 45 (1977), 8-12.

[24] Z. Zheng. The Petersson-Knopp indentity for the homogeneous Dedekind sums. J. Number Theory 57 (1996), 223-230.

\author{
Kazuhito Kozuka \\ Department of Mathematics \\ Miyakonojo National College of Technology \\ Miyakonojo \\ Miyazaki 885-8567 \\ Japan \\ (E-mail:k31k@cc.miyakonojo-nct.ac.jp)
}

